\title{
Uso das Sementes de Moringa Oleifera no Tratamento da Água
}

\section{Use of Moringa Oleifera Seeds in Water Treatment}

\author{
Marcia Kiyomi Koike, ${ }^{1,2}$ Akimi Kokanj Kochi, ${ }^{2}$ Denise Yamada Gomes Pinto ${ }^{2}$ \\ Laboratório de Emergências Clínicas - Departamento de Clínica Médica - Faculdade de Medicina da Universidade de São Paulo, ${ }^{1}$ São Paulo, Brasil \\ Programa de Pós-graduação em Ciências da Saúde - Instituto de Assistência ao Servidor Público Estadual de São Paulo (IAMSPE), ${ }^{2}$ São Paulo, \\ $S P-$ Brasil \\ Minieditorial referente ao artigo: Avaliação dos Efeitos Cardíacos de Lectina Solúvel em Água (WSMoL) de Sementes de Moringa Oleifera
}

A Moringa oleifera Lamarck $(\mathrm{MO})$ é uma planta da família das Moringaceae, nativa dos Himalaias e que se adaptou nos diversos continentes, sendo amplamente cultivada na Ásia, África e Américas. ${ }^{1}$ De crescimento rápido, a planta toda tem uma grande variedade de aplicações na dieta e na medicina popular.

No entanto, evidências científicas de suas propriedades começaram a surgir apenas no início de 2000. Em estudos experimentais in vitro ou ex-vivo, as folhas e sementes apresentaram diversos efeitos biológicos, como anti-inflamatórios e cicatrizantes, ${ }^{2}$ antitumorais, ${ }^{3}$ antidiabético, ${ }^{4}$ antioxidante ${ }^{4,5}$ e na função sexual. ${ }^{6,7}$

Devido suas propriedades de flotação e ação antimicrobiana, as sementes têm sido utilizadas na purificação da água. ${ }^{8}$ Trata-se de um método de baixo custo, que faz uso de recursos naturais e de fácil manuseio, que pode oferecer qualidade na água das comunidades pobres. As sementes podem adsorver poluentes como herbicidas, ${ }^{9}$ metais pesados, ${ }^{10}$ medicamentos ${ }^{11,12}$ e atuar como larvicidas e antimicrobianos natural. ${ }^{13}$

Entre os componentes presentes na semente de Moringa oleifera, a lectina solúvel em água (WSMoL) apresenta a propriedade de larvicida e ovicida do Aedes aegypti ${ }^{14} \mathrm{e}$ antinematódeos. ${ }^{15}$
Como todo conhecimento científico sobre a Moringa oleifera ainda está baseado em estudos experimentais, há necessidade de respeitar as etapas da pesquisa clínica para uso em humanos. Assim, em 2019, a Agência Nacional de Vigilância Sanitária (ANVISA) proibiu a fabricação, importação, comercialização, propaganda e distribuição de todos os alimentos que contenham Moringa oleifera (RESOLUÇÃO-RE No 1.478, DE 3 DE JUNHO DE 2019).

Da mesma forma, em relação ao uso das sementes para purificação de água, um estudo para se certificar da segurança também faz necessário. Neste contexto, nesta edição dos Arquivos Brasileiros de Cardiologia, Yurre et al. ${ }^{16}$ conduziram uma cuidadosa investigação sobre os efeitos cardiotóxicos da WSMoL de sementes de Moringa oleifera. Os autores avaliaram os possíveis efeitos celulares, estruturais, elétricos e funcionais no coração; bem como efeitos no metabolismo de carboidratos e no peso corporal. O estudo buscou testar sua hipótese nula e teve êxito em demonstrar, experimentalmente, a segurança de utilização da WSMoL por 21 dias, e estimula novos projetos de avaliação da segurança do uso de sementes de Moringa oleifera para a purificação da água para o uso humano.

\section{Palavras-chave}

Moringa Oleífera; Glicosídeos; Anti-Inflamatórios; Lectinas de Plantas; Segurança Hídrica.

Correspondência: Marcia Kiyomi Koike •

Universidade de São Paulo Faculdade de Medicina - LIM-51 -

Emergências clínicas

Av. Dr Arnaldo, 455 sala 3189. CEP 01246-903, São Paulo, SP - Brasil

E-mail: mkkoike17@gmail.com

DOI: https://doi.org/10.36660/abc.20200390 


\section{Referências}

1. Matic I, Guidi A, Kenzo M, Mattei M, Galgani A. Investigation of medicinal plants traditionally used as dietary supplements: A review on Moringa oleifera.J Public Health Afr. 2018 Dec 21;9(3):841.

2. Udupa SL, Udupa AL, Kulkarni DR. Studies on anti-inflammatory and wound healing properties of Moringa oleifera and Aegle marmelos. Fitoterapia. 1994;65:119-23.

3. Sadek KM, Abouzed TK, Abouelkhair R, Nasr S. The chemo-prophylactic efficacy of an ethanol Moringa oleifera leaf extract against hepatocellular carcinoma in rats. Pharmaceutical Biology. 2017;55(1):1458-66.

4. Gupta R, Mathur M, Bajaj VK, Katariya P, Yadav S, Kamal R,et al. Evaluation of antidiabetic and antioxidant activity of Moringa oleifera in experimental diabetes. J Diabetes. 2012;4(2):164-71.

5. Shaat AR, Sadek KM, El-Far AH, Nasr SM, El-Sayed Y. Evaluation of antioxidant and hepatoprotective activities of moringa (moringa oleifera) leaves in diabetic rabbits. Eur J Pharmac Med Res. 2017;4(7):154-62.

6. Sadek KM. Chemotherapeutic efficacy of an ethanolic Moringa oleifera leaf extract against chromium-induced testicular toxicity in rats. Andrologia. 2014;46:1047-54.

7. Prabsattroo T, Wattanathorn J, lamsaard S, Sompsat P, Sritragool O, Thukhummee W,et al. Moringa oleifera extract enhances sexual performance in stressed rats. J Zhejiang Univ Sci. 2015;16(3):179-90.

8. Nouhi S, Kwaambwa HM, Gutfreund P, Rennie AR. Comparative study of flocculation and adsorption behaviour of water treatment proteins from Moringa peregrina and Moringa oleifera seeds. Sci Rep. 2019;9(1):17945.

9. Cusioli LF, Bezerra CO, Quesada HB, Alves Baptista AT, Nishi L, Vieira MF, et al. Modified Moringa oleifera Lam. Seed husks as low-cost biosorbent for atrazine removal. Environ Technol. 2019 Aug 14:1-12.
10. Freitas $J \mathrm{H}$, de Santana KV, da Silva PM, de Moura MC, Coelho LC, do Nascimento AE, et al. Evaluation of Moringa oleifera Seed Lectin as a Metal Remover in Aqueous Solutions. Protein Pept Lett. $2016 ; 23(7): 645-9$.

11. Bagheri A, Abu-Danso E, Iqbal J, Bhatnagar A. Modified biochar from Moringa seed powder for the removal of diclofenac from aqueous solution.Environ Sci Pollut Res Int. 2020;27(7):7318-27.

12. Santos AF, Matos M, Sousa Â, Costa C, Nogueira R, Teixeira JA, et al. Removal of tetracycline from contaminated water by Moringa oleifera seed preparations. Environ Technol. 2016;37(6):744-51.

13. Moura MC, Trentin DS, Napoleão TH, Primon-Barros M, Xavier AS, Carneiro NP, et al. Multi-effect of the water-soluble Moringa oleifera lectin against Serratia marcescens and Bacillus sp.: antibacterial, antibiofilm and anti-adhesive properties.J Appl Microbiol. $2017 ; 123(4): 861-74$.

14. Silva LLS, Fernandes KM, Miranda FR, Silva SCC, Coelho LCBB, Navarro DMD, et al. Exposure of mosquito (Aedes aegypti) larvae to the water extract and lectin-rich fraction of Moringa oleifera seeds impairs their development and future fecundity. Ecotoxicol Environ Saf. 2019 Nov $15 ; 183: 109583$.

15. de Medeiros MLS, de Moura MC, Napoleão TH, Paiva PMG, Coelho LCBB, Bezerra ACDS, et al. Nematicidal activity of a water soluble lectin from seeds of Moringa oleifera. Int J Biol Macromol. 2018;108:782-9.

16. Yurre AR, Silva JD, Torres MK, Martins EL, Ramos IP, Lima da Silva WS, et al. Avaliação dos Efeitos Cardíacos de Lectina Solúvel em Água (WSMoL) de Sementes de Moringa Oleifera . Arq Bras Cardiol. 2020; 114(6):1029-1037. 